\title{
Rapidly progressing A/H1N1 (09) pandemic influenza virus infection in pregnancy
}

\author{
Celen $\mathrm{S}^{1}$, Ozyer $\mathrm{S}^{1}$, Unlu $\mathrm{S}^{2}$, Saygan $\mathrm{S}^{2}$, Uzunlar $\mathrm{O}^{1}$, Danısman $\mathrm{N}^{1}$, Mollamahmutoglu L ${ }^{1}$ \\ Department of Obstetrics and Gynecology, Zekai Tahir Burak Women's Health Education and Research Hospital, \\ Ankara, Turkey.sebnemsenozyer@yahoo.com
}

\begin{abstract}
We presented a pandemic H1N1 2009 influenza virus infection in two pregnant women who were referred to the intensive care unit (ICU) with a rapidly progressive disease course. Both women had unremarkable past medical history. The first woman had the symptoms of fever, cough, dyspnea and tachypnea lasting for four days. The second woman refused to take oseltamivir treatment. They were both referred to ICU with the diagnosis of pneumonia and acute respiratory distress syndrome. The second woman was intubated for two days in ICU. Both women showed a gradual improvement in their follow up and were discharged. Early admission, prompt diagnosis and early recourse to antiviral treatment gives better results in $\mathrm{H} 1 \mathrm{~N} 1$ infected pregnant women (Fig. 2, Ref. 10). Full Text in PDF www.elis.sk.

Key words: H1N1, influenza, pregnancy, intensive care unit.
\end{abstract}

Pandemic H1N1 2009 influenza virus infection caused a widespread outbreak of respiratory infection. The virus, which originated in Mexico, spread globally to nearly 191 countries and territories worldwide. The novel virus contained a unique combination of gene segments that were not identified before $(1,2)$.

The cases of the current pandemic presented with mild symptoms such as body aches, fatigue, chills, rhinorrhea, conjunctivitis, shortness of breath, headache and gastrointestinal (GI) symptoms. GI symptoms such as diarrhea and vomiting appeared more frequently than seasonal influenza. Pregnant women tend to present with fever, cough and/or sore throat besides these symptoms (3). They experienced shortness of breath more often than nonpregnant cases.

During both interpandemic seasonal influenza and previous pandemics, it was found that pregnant women and fetus were at an increased risk for complications. During the period of April 15, 2009 to June 16, 2009, CDC reported 45 deaths from H1N1 infection; six (13\%) of these individuals were pregnant (4). From April 23 to August 11, 2009, a total of $10 \%$ of the 1088 patients who were hospitalized with or died from 2009 H1N1 influenza, as reported to the California Department of Public Health (CDPH), were pregnant (5). The CDC had implemented an enhanced surveillance for infection with $\mathrm{H} 1 \mathrm{~N} 1$ in pregnant women because of the concerns about the severity of the disease during pregnancy (3).

'Department of Obstetrics and Gynecology, Zekai Tahir Burak Women's Health Education and Research Hospital, Ankara, Turkey, and ${ }^{2}$ Department of Infectious Diseases, Zekai Tahir Burak Women's Health Education and Research Hospital, Ankara, Turkey

Address for correspondence: S. Ozyer, MD, Birlik mah 406. sok 15/9 Cankaya, Ankara, Turkey.

Phone: +90.312.4950602, Fax: +90.312.3124931
In this report, we presented two pregnant women with pandemic H1N1 2009 influenza virus infection who were hospitalized in the three months of outbreak when the pandemic influenza cases peaked in our country.

Case 1

A 22-years-old woman at 16 weeks' gestation presented with the symptoms of fever, cough, sore throat, myalgia, dehydration, dyspnea and tachypnea, which was present for four days. She had also vaginal bleeding and pelvic pain for the last two days. Her body temperature was $39.6^{\circ} \mathrm{C}$, blood pressure was $90 / 60 \mathrm{mmHg}$, heart rate was 112 beats per minute (bpm) and respiratory rate was 25 per minute. Oxygen saturation level was $95 \%$ on room air on

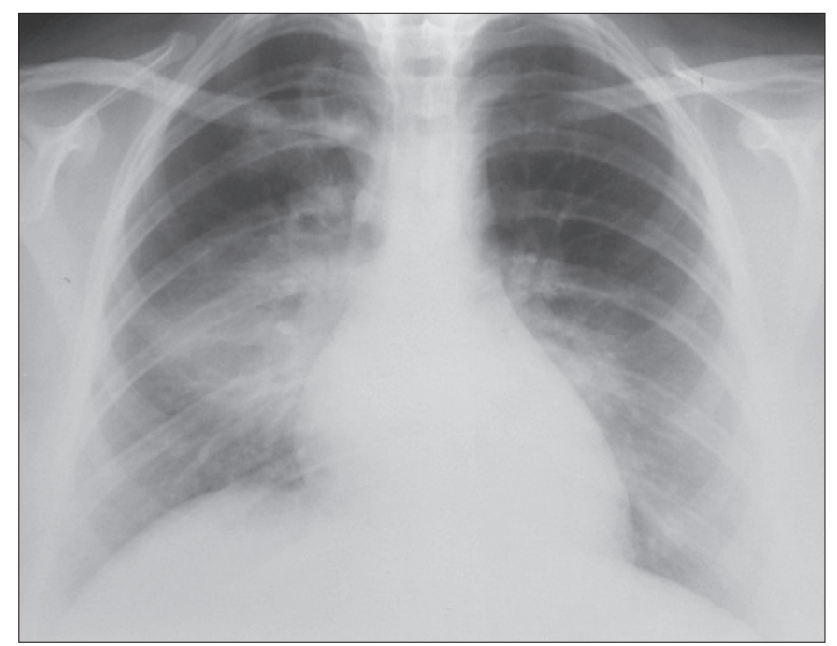

Fig. 1. Chest X-ray showing a bilateral diffuse infiltration. 
379-381

admission. The patient was a non-smoker and her past medical history was unremarkable. Chest auscultation revealed dullness, inspiratory crackles and rales. The patient was isolated from other pregnant patients for the evaluation of a probable H1N1 virus infection. Samples were taken for H1N1 virus testing from nose and throat. Vaginal bleeding was observed on pelvic examination. Ultrasonographic examination revealed an intrauterine pregnancy consistent with the gestational age. White blood cell count was $9 \times 10^{9} / \mathrm{L}$. Chest X-ray revealed a diffuse bilateral infiltration (Fig. 1 ). After an increase in her vaginal bleeding and rupture of membranes, the pregnancy ended with abortion. The morphologic appearance of the fetus was normal. Although she was started on empiric therapy of oseltamivir (Tamiflu, Roche, Turkey) (75 mg, twice daily, PO) and antibiotic treatment of sulbactam-ampicillin (Duocid, Pfizer, Turkey) (1 g, four times a day, i.v.) for the superimposed pneumonia, she had a rapidly progressive disease course. Oxygen saturation level decreased below $80 \%$. Arterial blood gas sampling revealed a $\mathrm{PaO}_{2}$ of $67 \mathrm{mmHg}$. She was transferred to the intensive care unit (ICU). During her follow up in ICU, she continued to take the treatment together with oxygen supplementation and ipratropium bromide (Combivent, Boehringer Ingelheim, Turkey) inhalation therapy. Her oxygen saturation level was held around $85 \%$ with nasal oxygen supplementation so she was not intubated. She clinically improved with this regimen. Laboratory results confirmed the H1N1 virus infection by a real-time reverse transcriptase PCR. She was discharged on hospital day 10.

\section{Case 2}

A 34-years-old woman at 36 weeks' of gestation was hospitalized with the diagnosis of an intrauterine growth restriction, oligohydramnios and flu-like symptoms. She presented with the symptoms of fever, cough, sore throat, myalgia and dehydration. Her body temperature was $39.2^{\circ} \mathrm{C}$, blood pressure was 100/60 mmHg, pulse was 112 beats per minute (bpm) and respiratory rate was 22 per minute on admission. Oxygen saturation level was $96 \%$ on room air. She was a non-smoker and her past medical history was unremarkable. She was isolated from other pregnant women and samples from nose and throat were taken to diagnose a possible H1N1 virus infection. White blood cell count was $6.8 \times 10^{9} / \mathrm{L}$ on admission. She was offered to take oseltamivir, which she refused to use. Ultrasound examination revealed an intrauterine pregnancy consistent with 33-35 weeks' gestation and oligohydramnios. External fetal monitoring, which revealed an absent variability at first, noted late decelerations on the fetal heart rate tracing soon after. Given this non reassuring fetal status, she delivered by an emergency cesarean. She delivered a female infant with the birth weight of $2320 \mathrm{~g}$ with Apgar scores of 8 at 1 st min and 10 at 5th min. The newborn was normal with no evidence of influenza infection. After the delivery, the patient developed dyspnea and tachypnea. Chest X-ray revealed diffuse bilateral infiltrations (Fig. 2). Oxygen saturation level decreased to $88 \%$. Arterial blood gas sampling revealed a $\mathrm{PaO}_{2}$ of $58.3 \mathrm{mmHg}$. She was referred to ICU. Laboratory results confirmed H1N1 virus infection by a real-time reverse transcriptase PCR. Oseltamivir was added 36 hours after

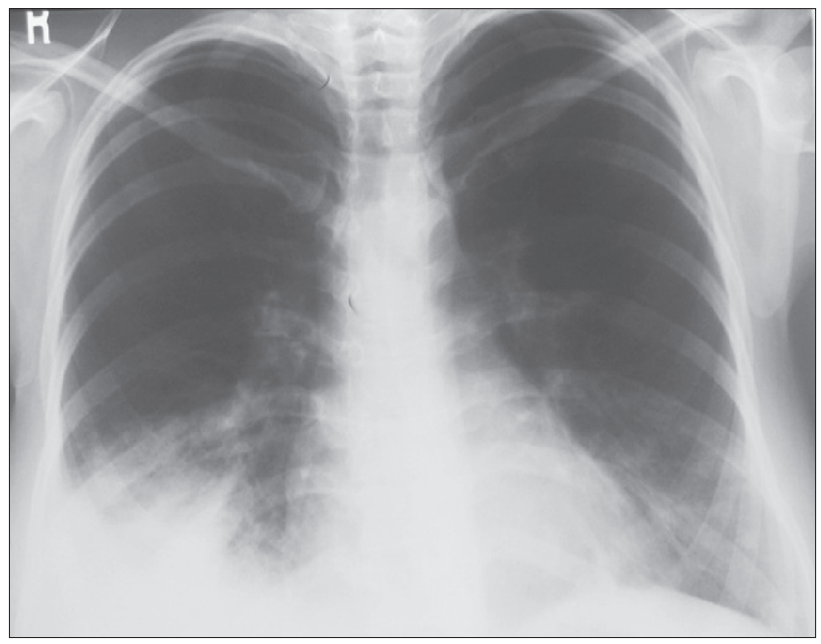

Fig. 2. Chest X-ray showing a pneumonic infiltration.

delivery to the empiric antibiotic therapy of sulbactam-ampicillin ( $1 \mathrm{~g}$, four times a day, PO) for the superimposed pneumonia after the patient's approval. Ipratropium bromide inhalation therapy was initiated. She remained intubated for two days in ICU. The patient showed a gradual improvement in her follow up and was discharged on hospital day 10.

\section{Discussion}

We present two different cases of H1N1 influenza virus infection with superimposed pneumonia and acute respiratory distress syndrome. We hospitalized 23 additional cases with laboratory confirmed H1N1 virus infection in our institution during three months of outbreak, from October to December, 2009. The diagnosis of influenza was confirmed by 'Swine Influenza PCR Testing Kit', which was prepared by Centres for Disease Control and Prevention (CDC) and distributed by World Health Organization (WHO). The kits were available from the National Influenza Center in Turkey. Although these two patients followed a rapidly progressive disease course, most of the pregnant women exhibited mild disease process with a stable course. In a recent study involving large series of pregnant and postpartum patients who were hospitalized with or died from 2009 H1N1 influenza, $95 \%$ of the pregnant patients were infected in the second or third trimester, and almost one fifth required intensive care (6).

The novel H1N1 2009 influenza virus is sensitive to neuraminidase inhibitors oseltamivir and zanamivir. The benefit of treatment with these antiviral drugs outweighs its theoretical risk. Current CDC guidance suggests that antiviral treatment is recommended for groups at high risk for influenza complications from infection with H1N1 2009 influenza virus including pregnant women. The CDC recommends a prompt antiviral treatment of pregnant women with suspected or confirmed 2009 H1N1 influenza, ideally within 48 hours after symptom onset (7). Because of its systemic activity, the drug of choice for pregnant women is oseltamivir. It can reduce mortality in patients even when started more than 48 hours after the onset of symptoms (4). The first case in this report had symptoms 
for four days and the second case refused to take antiviral therapy because of concerns about the fetus. These factors caused a delay in the initiation of the treatment and might be responsible for the rapid progression of the disease. Early admission and prompt initiation of the antiviral therapy could have resulted in a shorter recovery period and better outcome in these cases.

Louie et al reported a continued high risk immediately after pregnancy in their study of H1N1 virus infection in pregnant, non pregnant and postpartum women (6). A variety of cardiac, respiratory, hormonal, and immunologic changes that occur during pregnancy may contribute to an increased risk of influenza-related morbidity and mortality among pregnant women (8). Although it is not known for how long after delivery these changes persist, some of them (i.e., immunologic alterations) might persist longer than others (e.g., decreased lung capacity due to uterine compression). In light of these emerging data, the CDC recently issued the revised guidelines, recommending a prompt initiation of antiviral treatment in patients with suspected or confirmed influenza up to two weeks after delivery (7).

The CDC has listed pregnant women as a priority group for vaccination, along with children and health care workers (9). The pregnant women are at a higher risk for complications and can also potentially provide protection to infants who cannot be vaccinated via the transfer of maternal antibodies to the fetus. On September 15,2009 , four influenza vaccine manufacturers received the approval from the Food and Drug Administration (FDA) for their novel influenza A(H1N1) 2009 monovalent vaccines. Since 2009, H1N1 monovalent vaccine is manufactured according to the same processes that are used for the seasonal influenza vaccine, its safety profile among pregnant women is expected to be similar to that of the seasonal influenza vaccine, which has consistently been shown to be safe during pregnancy (10).

Women with suspected or confirmed influenza who are pregnant or who have delivered within the previous two weeks should receive an aggressive antiviral treatment and undergo close monitoring. Timely medical attention and early recourse to antiviral therapy is associated with a better outcome in H1N1 affected pregnant women. Fever and low oxygen saturation levels should alert the clinician about the development of a complication such as pneumonia and respiratory distress. Since pregnant women and their fetuses require an intensive care and monitoring, critically ill pregnant and postpartum women should be hospitalized in centres including the neonatal intensive care units, which can provide a higher level of care for both mother and the newborn.

\section{References}

1. Novel Swine-Origin Influenza A (H1N1) Virus Investigation Team. Emergence of a novel swine-origin influenza A (H1N1) virus in humans. N Engl J Med 2009; 360: 2605-2615. (Erratum, N Engl J Med 2009; 361: 102.)

2. Garten RJ, Davis CT, Russell CA, et al. Antigenic and genetic characteristics of swine-origin 2009 A (H1N19 influenza viruses circulating in humans. Science 2009; 325: 197-201.

3. Satpathy HK, Lindsay M, Kawwass JF. Novel H1N1 virus infection and pregnancy. Postgrad Med 2009; 121 (6): 106-112.

4. Jamieson DJ, Honein MA, Rasmussen SA, et al. H1N1 2009 influenza virus infection during pregnancy in the USA. Novel Influenza A (H1N1) Pregnancy Working Group. Lancet 2009; 374 (9688): 451-458.

5. Louie JK, Acosta M, Winter K, et al. Factors associated with death or hospitalization due to pandemic 2009 influenza A (H1N1) infection in California. JAMA 2009; 302: 1896-1902.

6. Louie JK, Acosta M, Jamieson DJ, Honein MA; California Pandemic (H1N1) Working Group. Severe 2009 H1N1 influenza in pregnant and postpartum women in California. N Engl J Med 2010; 362 (1): 27-35.

7. Centers for Disease Control and Prevention. Updated interim recommendations for obstetric health care providers related to use of antiviral medications in the treatment and prevention of influenza for the 2009-2010 season. (Accessed December 16, 2009, at http://www.cdc.gov/ h1n1flu/ pregnancy/antiviral_messages.htm.)

8. Jamieson DJ, Theiler RN, Rasmussen SA. Emerging infections and pregnancy. Emerg Infect Dis 2006; 12: 1638-1643.

9. Chan KH, Lai ST, Poon LL, Guan Y, Yuen KY, Peiris JS. Analytical sensitivity of rapid influenza antigen detection tests for swine-origin influenza virus (H1N1). J Clin Virol 2009; 45 (3): 205-207.

10. Tamma PD, Ault KA, Del Rio C, Steinhoff MC, Halsey NA, Omer SB. Safety of influenza vaccination during pregnancy. Am J Obstet Gynecol 2009 (Epub ahead of print). 